\title{
Propiedades Psicométricas de la Escala de Autoeficacia Generalizada en Cuidadores no Profesionales
}

\author{
Psychometric Properties of the Generalized Self-Efficacy Scale in Non-Professional \\ Caregivers
}

\author{
Vanessa Blanco ${ }^{1}$, Fernando L. Vázquez ${ }^{2}$, María Adelina Guisande ${ }^{3}$, María Teresa Sánchez y \\ Patricia Otero ${ }^{5}$
}

\section{Resumen}

El objetivo de este estudio fue analizar las propiedades psicométricas de la Escala de Autoeficacia Generalizada (GSES) en cuidadores no profesionales. Tres psicólogas entrenadas recogieron los datos sociodemográficos y de la situación de cuidado, la GSES, la CES-D, el ATQ-30 y la EPQ-R en 592 cuidadores $(87.16 \%$ mujeres; edad media $=55.41$ años). Posteriormente, dos expertos en evaluación y diagnóstico valoraron el diagnóstico de episodio depresivo mayor, usando el SCID-5. La muestra total se dividió aleatoriamente en dos grupos para el análisis factorial exploratorio (AFE) y el análisis factorial confirmatorio (AFC). La consistencia interna fue de $\alpha=.90$. Un único factor explicó el $74.17 \%$ de la varianza total. Se encontraron correlaciones de la autoeficacia con extraversión, neuroticismo, sintomatología depresiva y pensamientos automáticos negativos. La GSES discriminó entre cuidadores deprimidos y no deprimidos clasificando correctamente el $68 \%$ de los casos. $\leq 28$ resultó un punto de corte adecuado para discriminar los cuidadores con o sin depresión. La GSES resultó un instrumento fiable y válido para evaluar la autoeficacia generalizada en cuidadores no profesionales.

Palabras clave: autoeficacia, GSES, cuidadores, propiedades psicométricas, análisis factorial confirmatorio

\begin{abstract}
The aim of this study was to analyse the psychometric properties of the Generalized Self-Efficacy Scale (GSES) in non-professional caregivers. Three trained psychologists collected the sociodemographic data and information on the situation of care, GSES, CES-D, ATQ-30 and EPQ-R of 592 non-professional caregivers ( $87.16 \%$ women, average age $=55.41$ years $)$. Subsequently, two experts in assessment and diagnosis assessed a major depressive episode diagnosis using the SCID-5. The total sample was randomly divided into two groups for exploratory factor analysis (EFA) and confirmatory factor analysis (CFA). The internal consistency was $\alpha=.90$. A single factor explained $74.17 \%$ of the total variance. Self-efficacy was correlated with extraversion, neuroticism, depressive symptomatology, and negative automatic thoughts. The GSES discriminated between depressed and non-depressed caregivers by correctly classifying $68 \%$ of the cases. The value of $\leq 28$ on the GSES was determined to be an appropriate cut-off point to discriminate between caregivers with or without depression. The GSES is a reliable and valid instrument to assess the generalized self-efficacy of non-professional caregivers.
\end{abstract}

Keywords: self-efficacy, GSES, caregivers, psychometric properties, confirmatory factor analysis

\footnotetext{
${ }^{1}$ Doctor, Profesora Contratada Interina por Sustitución, Universidad de Santiago de Compostela. Departamento de Psicología Evolutiva y de la Educación. Facultad de Psicología, Universidad de Santiago de Compostela, Campus Vida, 15782. Santiago de Compostela, España. Correo: vanessa.blanco@usc.es. Tel.: +34881813744. (Correspondencia)

${ }^{2}$ Doctor, Profesor Titular de Universidad, Universidad de Santiago de Compostela. Departamento de Psicología Clinica y Psicobiología. Facultad de Psicología, Universidad de Santiago de Compostela, Campus Vida, 15782. Santiago de Compostela, España. Correo: fernandolino.vazquez@usc.es. Tel.: +34881813705.

${ }^{3}$ Doctor, Profesor Contratado Doctor, Universidad de Santiago de Compostela. Departamento de Psicología Evolutiva y de la Educación. Facultad de Psicología, Universidad de Santiago de Compostela, Campus Vida, 15782. Santiago de Compostela, España. Correo: mariaadelina.guisande@usc.es. Tel.: +34881813919.

${ }^{4}$ Doctor, Profesor Titular de Universidad, Universidad de Santiago de Compostela. Departamento de Psicología Evolutiva y de la Educación. Facultad de Ciencias de la Educación, Universidad de Santiago de Compostela. Campus Norte, 15782, Santiago de Compostela, España. Correo: mariateresa.sanchez@usc.es. Tel.: +34881812109.

${ }^{5}$ Doctor, Profesora Contratada Interina por Sustitución, Universidad de A Coruña. Departamento de Psicología, Facultad de Ciencias de la Educación, Universidad de A Coruña. Campus de Elviña, 15071, A Coruña, España. Correo: patricia.otero.otero@udc.es. Tel.: +34981167000, Ext: 4683.

Revista Iberoamericana de Diagnóstico y Evaluación - e Avaliação Psicológica. RIDEP · N52 · Vol.3 · 115-127 · 2019

ISSN: 1135-3848 print /2183-6051online
} 


\section{Introducción}

El envejecimiento de la población conducirá en los próximos años a un enorme incremento en la proporción de personas que necesitarán cuidados debido a distintos grados de dependencia en todo el mundo (World Health Organization [WHO], 2015). En Europa, en un $80 \%$ de los casos, esta atención es prestada de manera no remunerada por familiares o allegados, denominados cuidadores no profesionales (Hoffmann \& Rodrigues, 2010). A menudo, su salud se ve menoscabada por el estrés asociado a la situación de cuidado (Litzelman et al., 2014) incrementando su riesgo de malestar emocional, especialmente depresión, con una prevalencia de $8.9 \%$ en esta población (Torres et al., 2015).

Sin embargo, existen distintos modos de afrontar el cuidado (p.ej., De Andrés-Jiménez \& Limiñana-Gras, 2014), y muchos cuidadores no desarrollan malestar emocional y son capaces de tener experiencias positivas a pesar del estrés (p.ej., Domínguez-Guedea, 2016). De acuerdo con el modelo teórico de Pearlin, Mullan, Semple, \& Skaff (1990), existen cuatro dominios implicados en el proceso de estrés del cuidado: (a) los antecedentes y el contexto, (b) los estresores, (c) los mediadores y (d) los resultados. Entre los potenciales mediadores del estrés, la autoeficacia podría explicar parte de la variabilidad para manejar las demandas crónicas que implica el cuidado, constituyendo un factor protector contra el estrés y la sobrecarga (Koerner, Kenyon, \& Shirai, 2009).

La autoeficacia ha sido definida como la creencia de una persona sobre su capacidad para realizar una tarea específica y movilizar la motivación, los recursos cognitivos y los cursos de acción necesarios para satisfacer demandas situacionales determinadas (Bandura, 1977; Bandura \& Wood, 1989), mientras que la autoeficacia generalizada se refiere a la confianza global en la propia capacidad de afrontamiento a través de un amplio rango de situaciones demandantes o novedosas (Schwarzer \& Jerusalem, 1995).

Aunque existen pocos estudios sobre autoeficacia en cuidadores, se ha encontrado que aquellos con mayor autoeficacia son menos vulnerables al estrés (Contador, Fernández-Calvo,
Palenzuela, Miguéis, \& Ramos, 2012); tienen un pensamiento más positivo, más control del afecto negativo, mayor motivación (Semiatin \& O’Connor, 2012) y mejor calidad de vida relacionada con la salud (Crellin, Orrell, McDermott, \& Charlesworth, 2014); y eligen tareas más desafiantes, se plantean objetivos más elevados, son más persistentes y tienen mayor compromiso con sus metas (Cheng, Lam, Kwok, $\mathrm{Ng}$, \& Fung, 2013). Además, una mayor autoeficacia tiene también un impacto positivo sobre la calidad del cuidado y la persona en situación de dependencia, relacionándose con un menor deseo de ingresarla en una institución residencial, potente predictor de una futura institucionalización (Gallagher et al., 2011). Por el contrario, aquellos cuidadores con autoeficacia baja experimentan más estados psicológicos negativos, como depresión o ansiedad (López, Romero-Moreno, Márquez-González, \& Losada, 2012); la identificación de estos cuidadores con baja autoeficacia permitiría la implementación de programas de promoción de la autoeficacia, que han obtenido resultados positivos en estudios previos con esta población (p.ej., Ducharme, Lévesque, Gendron, \& Legault, 2001; Savundranayagam, Montgomery, Koslosky, \& Little, 2011). Estos hallazgos sugieren la importancia de evaluar la autoeficacia en cuidadores no profesionales.

A pesar de esto, pocas escalas de autoeficacia han sido validadas para personas cuidadoras y la mayoría obtuvieron puntuaciones bajas $\mathrm{o}$ moderadas en los criterios de calidad, con falta de información sobre los efectos suelo y techo, entre otros (Stansfeld et al., 2017). Además, consideran la autoeficacia como un constructo específico de dominio pero, en nuestro conocimiento, no se han desarrollado o validado instrumentos de evaluación de la autoeficacia general en la población de cuidadores.

Entre los instrumentos más empleados para evaluar la autoeficacia general se encuentra la Escala de Autoeficacia Generalizada (GSES; Schwarzer \& Jerusalem, 1995). La versión original de Jerusalem y Schwarzer constaba de 20 ítems, posteriormente se redujo a 10 (Schwarzer, 1993) y fue traducida a 28 idiomas. Esta última versión se validó con datos de 1.660 adultos alemanes, desde estudiantes a personas mayores 
(Schwarzer, 1993), encontrando que era un instrumento fiable que evaluaba un constructo unidimensional y universal (Schwarzer \& Jerusalem, 1995). Esto fue corroborado en un trabajo en el que se analizaron datos de 19.120 estudiantes y profesionales de más de 25 países (Bélgica, Canadá, Costa Rica, España y EEUU, entre otros) (Scholz, Doña, Sud, \& Schwarzer, 2002); además, la muestra de 943 universitarios costarricenses se utilizó para analizar las propiedades psicométricas de la primera versión española de este instrumento (Baessler \& Schwarzer, 1996). En un trabajo posterior con 8.796 estudiantes, profesores y personas inmigrantes de cinco países se encontraron asociaciones positivas de la autoeficacia con autoestima y negativas con depresión (Luszczynska, Gutiérrez-Doña, \& Schwarzer, 2005). Asimismo, las propiedades psicométricas de la GSES han sido analizadas en diversas poblaciones como adultos noruegos (Leganger, Kraft, \& Røysamb, 2000); adolescentes (Espada, Gonzálvez, Orgilés, Carballo, \& Piqueras, 2012), universitarios (Sanjuán, Pérez, \& Bermúdez, 2000) y pacientes psiquiátricos ambulatorios españoles (De las Cuevas \& Peñate, 2015); o población general y personas en situación de baja laboral de Suecia (Löve, Moore \& Hensing, 2012). Sin embargo, no existen datos sobre las propiedades psicométricas de la GSES en cuidadores. El objetivo del presente estudio fue analizar las propiedades psicométricas de la versión española de la GSES en cuidadores no profesionales.

\section{Método}

\section{Participantes}

Se empleó un diseño transversal. La muestra fue extraída a través de un muestreo aleatorio simple entre los 18,410 cuidadores no profesionales de personas en situación de dependencia de la comunidad autónoma de Galicia (España) inscritos en el registro oficial de cuidadores de la Consellería de Trabajo y Bienestar de la Xunta de Galicia.

Se incluyó a aquellos sujetos que: (a) eran cuidadores principales de una persona en situación de dependencia reconocida oficialmente, (b) vivían con ella y $\quad$ (c) proporcionaron consentimiento informado. Se excluyó a aquellos que: (a) presentaban dificultades en la comunicación (p.ej., no saber leer ni escribir) o cualquier condición que hiciese imposible la evaluación (p.ej., discapacidad intelectual, deficiencia visual severa) o (b) habían recibido tratamiento psicológico o farmacológico en los dos últimos meses.

Para la estimación del tamaño muestral se tuvieron en cuenta el número de ítems que definían cada factor y la comunalidad de los ítems, considerando el peor escenario, o condición mínima, consistente en un número de variables por factor de 3 ítems y comunalidades bajas (en torno a .30) (Lloret- Segura, FerreresTraver, Hernández-Baeza, \& Tomás-Marco, 2014), determinándose que era necesaria una muestra de más de 500 sujetos para conseguir estimaciones precisas (Hogarty, Hines, Kromrey, Ferron, \& Mumford, 2005). La tasa de respuesta fue del $98.18 \%$. De las 603 personas invitadas a participar, 11 no cumplimentaron adecuadamente la evaluación y fueron eliminadas de los análisis, quedando una muestra final de 592 sujetos (87.16\% mujeres) de edades comprendidas entre los 25 y los 86 años ( $M=55.41$ años; $D T=11.92)$.

Todos los participantes fueron informados de la naturaleza del estudio y dieron su consentimiento informado. La participación fue voluntaria, sin incentivos económicos o de otro tipo. El estudio se realizó de acuerdo a la Declaración de Helsinki y fue aprobado por el Comité de Bioética de la Universidad de Santiago de Compostela.

\section{Instrumentos}

Cuestionario de las Características del Cuidador y de la Situación de Cuidado. Se trata de un instrumento elaborado ad hoc para recoger variables sociodemográficas del cuidador (sexo, edad, estado civil, clase social, nivel educativo) y de la situación de cuidado (parentesco con la persona cuidada, sexo, edad y enfermedad, tiempo en años cuidándola y horas diarias dedicadas al cuidado).

Escala de Autoeficacia Generalizada (Generalized Self-Efficacy Scale [GSES]; Schwarzer \& Jerusalem, 1995; versión española de Baessler \& Schwarzer, 1996). Esta escala 
autoinformada consta de 10 ítems, con un formato de respuesta tipo Likert de 4 puntos, de 1 (incorrecto) a 4 (cierto), con un rango de puntuaciones de 10 a 40, donde mayor puntuación indica mayor autoeficacia. La consistencia interna de la versión original en alemán osciló de .82 a .93 .

Además, con el objeto de analizar la validez convergente y criterial con la GSES se administraron los siguientes instrumentos:

Entrevista Clínica Estructurada para los Trastornos del DSM-5, Versión Clínica (The Structured Clinical Interview for DSM-5® Clinician Version [SCID-5-CV]; First, Williams, Karg, \& Spitzer, 2015). Entrevista semiestructurada que proporciona diagnósticos del DSM-5 y debe ser administrada por un psicólogo. Las preguntas se corresponden los criterios del DSM-5, que deben ser calificados como presentes o ausentes. Para la evaluación se utilizó el módulo correspondiente al episodio depresivo mayor. La fiabilidad entre jueces (Kappa) oscila entre $.70 \mathrm{y}$ 1.00 .

Escala de Depresión del Centro para Estudios Epidemiológicos (Center for Epidemiological Studies Depression Scale [CES-D]; Radloff, 1977; versión española de Vázquez, Blanco, \& López, 2007). Escala autoinformada de 20 ítems que proporciona una puntuación continua de la sintomatología depresiva durante la última semana. El rango va de 0 a 60 , donde mayor puntuación corresponde a mayor sintomatología depresiva. La consistencia interna (alfa de Cronbach) para la versión española fue .89 .

Cuestionario de Pensamientos Automáticos (Automatic Thoughts Questionnaire [ATQ-30]; Hollon \& Kendall, 1980; versión española de Otero, Vázquez, Blanco, \& Torres, 2017). Cuestionario de 30 ítems que evalúa la frecuencia con que una serie de pensamientos han surgido súbitamente en la cabeza del evaluado durante la última semana. El rango de puntuación oscila entre 30 y 150 , donde mayor puntuación corresponde a más pensamientos negativos. La consistencia interna (alfa de Cronbach) de la versión española fue .96 .

Cuestionario de Personalidad de Eysenck Revisado-Abreviado (Eysenck Personality Questionnaire Revised-Abbreviated [EPQR-A]; Francis, Brown, \& Philipchalk, 1992; versión española de Sandín, Valiente, Chorot, Olmedo, \& Santed, 2002). Cuestionario autoadministrado de 24 ítems y 4 subescalas; tres miden rasgos de personalidad (Neuroticismo, Extraversión, Psicoticismo) y una cuarta la tendencia a responder de manera socialmente deseable (Sinceridad). Las puntuaciones en cada subescala oscilan entre 0 y 6 y mayor puntuación indica mayor presencia del rasgo. La consistencia interna (alfa de Cronbach) para cada subescala es: Neuroticismo =.78, Extraversión =.74, Psicoticismo $=.63$, y Sinceridad $=.54$ (Sandín, Valiente, Olmedo, Chorot, \& Santed, 2002).

\section{Procedimiento}

Los cuidadores fueron contactados a través de una carta invitándoles a formar parte del estudio y solicitándoles que devolviesen una tarjeta postal sellada si no deseaban participar. Aquellos que no la devolvieron fueron contactados, se realizó una breve descripción del estudio y, a los interesados, se les invitó a participar. Para minimizar la pérdida de sujetos, se siguieron las estrategias recomendadas por Hulley, Cummings y Newman (2013) que incluyeron enviar recordatorios de la fecha de evaluación, evitar procedimientos de recogida de información invasivos y presentar el estudio de manera interesante. Tres psicólogas entrenadas recogieron de forma heteroadministrada los datos sociodemográficos y de la situación de cuidado y aplicaron de forma autoadministrada la GSES, la CES-D, el ATQ-30 y el EPQR-A. El entrenamiento de las evaluadoras fue realizado por un experto en evaluación psicológica y consistió en dos sesiones de 90 minutos que incluían charlas y juego de roles. Posteriormente se realizó una prueba piloto, entrevistando cada evaluadora a 5 cuidadores de características similares a los que participaron en el estudio. Estas pruebas se grabaron para perfeccionar la evaluación y valorar el grado de adherencia al protocolo. Se proporcionó feedback hasta que lograron realizar adecuadamente la recogida de información.

Con posterioridad, dos expertos en evaluación y diagnóstico con más de 20 años de experiencia enmascarados a los resultados de los instrumentos autoadministrados valoraron la idoneidad del diagnóstico de episodio depresivo mayor usando 
la SCID-5. La duración de cada evaluación fue de aproximadamente 60 minutos.

\section{Análisis de datos}

Se calcularon frecuencias, porcentajes, medias y desviaciones típicas de las variables sociodemográficas y de la situación de cuidado, las puntuaciones de autoeficacia y los ítems de la GSES, los síntomas depresivos, pensamientos automáticos negativos, rasgos de personalidad y diagnóstico de depresión, para la muestra total y para las dos submuestras. Aquellos que no cumplimentaron adecuadamente los instrumentos de evaluación se eliminaron de los análisis. Para analizar las diferencias en autoeficacia en función de las características sociodemográficas y de la situación de cuidado se utilizaron pruebas $t$ de Student, ANOVA y correlaciones de Pearson, tanto para la muestra total como para las dos submuestras.

Para analizar la consistencia interna de la GSES se calcularon correlaciones de Pearson de sus ítems entre sí y de las puntuaciones de cada ítem con la puntuación total corregida, así como el coeficiente $\alpha$ de Cronbach, sobre la muestra total. Asimismo, con el objetivo de analizar la estructura factorial de la GSES, se llevaron a cabo dos procedimientos, análisis factorial exploratorio (AFE) y análisis factorial confirmatorio (AFC), con dos submuestras distintas. Para ello se dividió la muestra de este estudio en dos submuestras seleccionando al azar el $50 \%$ de los casos. A continuación, el AFE se llevó a cabo en una de las submuestras y el AFC en la otra. En concreto, para explorar las dimensiones subyacentes de la escala se realizó un AFE sobre la primera submuestra empleando un procedimiento basado en matrices de correlaciones policóricas, cuya pertinencia se determinó empleando la prueba de Mardia (1970). La medida de adecuación de la muestra se realizó mediante el índice de KaiserMeyer-Olkin (KMO) y la matriz de correlaciones identidad se contrastó empleando el test de esfericidad de Bartlett. El número de factores se determinó a través del análisis paralelo de Implementación óptima, basado en el análisis factorial de rango mínimo (PA-MRFA; Timmerman \& Lorenzo-Seva, 2011), la bondad de ajuste de los datos al modelo se estableció a través del porcentaje de varianza total explicada por los factores (Abad, Olea, Ponsoda, \& García, 2011; Lorenzo-Seva, \& Ferrando, 2006).

Para verificar la estructura factorial se realizó un análisis factorial confirmatorio (AFC). Se calculó la bondad del ajuste con el criterio de cuadrados mínimos ponderados, empleando (a) el estadístico $\chi^{2}$; (b) la ratio $\chi^{2} / \mathrm{df}$; (c) la raíz cuadrada media estandarizada (SRMR); (d) el error cuadrático medio de aproximación (RMSEA); (e) el índice de bondad de ajuste (GFI); (f) el índice de bondad de ajuste ajustado (AGFI); (g) el índice de ajuste normalizado (NFI); y (h) el índice de ajuste comparativo (CFI). Se consideran indicativos de un buen ajuste la ausencia de significación en $\chi^{2}$ y los valores: (a) SRMR <.08; (b) RMSEA <.06 (Hu \& Bentler, 1999); (c) GFI o AGFI >.90 (Baumgartner \& Homburg, 1996); (d) NFI o CFI próximos a .95 (Hu \& Bentler, 1999).

Sobre la muestra total, en relación a la validez convergente, se calcularon correlaciones de Pearson entre la GSES y las subescalas de neuroticismo y extraversión de la EPQ-R. Para analizar la validez discriminante se calculó la correlación entre la GSES y la subescala de psicoticismo de la EPQ-R. Con respecto a la validez criterial, se analizaron las correlaciones de Pearson de la GSES con la sintomatología depresiva y los pensamientos automáticos negativos, así como la $t$ de Student para muestras independientes para sufrir o no depresión. Además, se calcularon la sensibilidad, especificidad, valor predictivo positivo (VPP) y valor predictivo negativo (VPN). De manera complementaria se llevó a cabo un análisis de curva ROC (Receiver's Operating Characteristics) con el objetivo de determinar el punto de corte óptimo. Los análisis se realizaron con los programas SPSS (versión 21.0), FACTOR 8 (Lorenzo-Seva \& Ferrando, 2012), y LISREL (versión 8.8).

\section{Resultados}

\section{Perfil de la muestra}

El $87.16 \%$ de los cuidadores fueron mujeres, con una edad media de 55.41 años $(D T=11.92)$ y un rango entre 25 y 86 años. La mayoría (72.13\%) tenían pareja, pertenecían a clase social alta o 
Tabla 1. Características Sociodemográficas, de Cuidado y Clínicas de la Muestra (n=592)

\begin{tabular}{|c|c|c|c|c|c|c|}
\hline \multirow[t]{2}{*}{ Variables } & \multicolumn{2}{|c|}{$\begin{array}{l}\text { Muestra total } \\
\quad(\mathrm{n}=592)\end{array}$} & \multicolumn{2}{|c|}{$\begin{array}{l}\text { Submuestra AFE } \\
\qquad(n=296)\end{array}$} & \multicolumn{2}{|c|}{$\begin{array}{c}\text { Submuestra AFC } \\
\quad(n=296)\end{array}$} \\
\hline & $n$ & $\%$ & $n$ & $\%$ & $n$ & $\%$ \\
\hline \multicolumn{7}{|l|}{ Sexo } \\
\hline Hombre & 76 & 12.84 & 40 & 13.51 & 36 & 12.16 \\
\hline Mujer & 516 & 87.16 & 256 & 86.49 & 260 & 87.84 \\
\hline \multicolumn{7}{|l|}{ Edad } \\
\hline$M(D T)$ & 55.41 & $(11.92)$ & 56.26 & (11.74) & 54.55 & (12.05) \\
\hline \multicolumn{7}{|l|}{ Estado civil } \\
\hline Sin pareja & 165 & 27.87 & 78 & 26.35 & 87 & 29.39 \\
\hline Con pareja & 427 & 72.13 & 218 & 73.65 & 209 & 70.61 \\
\hline \multicolumn{7}{|l|}{ Clase social } \\
\hline Baja/media-baja & 287 & 48.48 & 138 & 46.62 & 149 & 50.34 \\
\hline Media/media-alta & 305 & 51.52 & 158 & 53.38 & 147 & 49.66 \\
\hline \multicolumn{7}{|l|}{ Nivel educativo } \\
\hline Sabe leer y escribir & 86 & 14.53 & 44 & 14.87 & 42 & 14.19 \\
\hline Primaria & 319 & 53.88 & 152 & 51.35 & 167 & 56.42 \\
\hline Secundaria/Universitario & 187 & 31.59 & 100 & 33.78 & 87 & 29.39 \\
\hline \multicolumn{7}{|l|}{ Parentesco con la persona cuidada } \\
\hline Padre/madre & 221 & 37.33 & 100 & 33.79 & 121 & 40.88 \\
\hline Cónyuge & 67 & 11.32 & 31 & 10.47 & 36 & 12.16 \\
\hline Hijo/a & 171 & 28.88 & 96 & 32.43 & 75 & 25.34 \\
\hline Otros familiares & 122 & 20.61 & 63 & 21.28 & 59 & 19.93 \\
\hline Otros no familiares & 11 & 1.86 & 6 & 2.03 & 5 & 1.69 \\
\hline \multicolumn{7}{|l|}{ Sexo de la persona cuidada } \\
\hline Hombre & 220 & 37.16 & 115 & 38.85 & 105 & 35.47 \\
\hline Mujer & 372 & 62.84 & 181 & 61.15 & 191 & 64.53 \\
\hline \multicolumn{7}{|l|}{ Edad de la persona cuidada } \\
\hline$M(D T)$ & 63.32 & (31.17) & 60.86 & $(32.37)$ & 65.78 & $(29.78)$ \\
\hline \multicolumn{7}{|l|}{ Enfermedad de la persona cuidada } \\
\hline Demencia & 178 & 30.07 & 82 & 27.71 & 96 & 32.43 \\
\hline $\begin{array}{l}\text { Trastornos mentales, neurológicos, } \\
\text { daño cerebral }\end{array}$ & 167 & 28.21 & 87 & 29.39 & 80 & 27.03 \\
\hline $\begin{array}{l}\text { Enfermedades del sistema } \\
\text { osteomuscular, tejido conjuntivo, } \\
\text { cardiovascular y respiratorio }\end{array}$ & 154 & 26.01 & 72 & 24.32 & 82 & 27.70 \\
\hline Otros & 93 & 15.71 & 55 & 18.58 & 38 & 12.84 \\
\hline \multicolumn{7}{|l|}{ Tiempo cuidando (años) } \\
\hline$M(D T)$ & 13.23 & (9.44) & 13.57 & $(9.65)$ & 12.89 & $(9.24)$ \\
\hline \multicolumn{7}{|l|}{ Horas diarias dedicadas al cuidado } \\
\hline \multicolumn{7}{|l|}{ Episodio depresivo mayor } \\
\hline No & 498 & 84.12 & 253 & 85.47 & 245 & 82.77 \\
\hline Sí & 94 & 15.88 & 43 & 14.53 & 51 & 17.23 \\
\hline \multicolumn{7}{|l|}{ Sintomatología depresiva } \\
\hline$M(D T)$ & 18.19 & (11.37) & 17.94 & $(11.36)$ & 18.45 & $(11.39)$ \\
\hline \multicolumn{7}{|l|}{ Pensamientos automáticos negativos } \\
\hline$M(D T)$ & 49.66 & (20.99) & 49.78 & $(22.00)$ & 49.53 & (19.96) \\
\hline \multicolumn{7}{|l|}{ Neuroticismo } \\
\hline$M(D T)$ & 3.01 & $(1.95)$ & 2.99 & (1.96) & 3.03 & (1.94) \\
\hline \multicolumn{7}{|l|}{ Extraversión } \\
\hline$M(D T)$ & 3.65 & $(1.87)$ & 3.65 & $(1.87)$ & 3.66 & $(1.88)$ \\
\hline \multicolumn{7}{|l|}{ Psicoticismo } \\
\hline$M(D T)$ & 1.26 & (.99) & 1.29 & $(1.00)$ & 1.22 & (.99) \\
\hline
\end{tabular}


Tabla 2. Medias y Desviaciones Típicas (DT) de los Ítems, Frecuencias de las Puntuaciones y Correlación Elemento-Total Corregida $\left(\mathrm{r}^{\text {tot }}\right)$ para Cada Ítem $(\mathrm{n}=592)$

\begin{tabular}{lccccccc}
\hline \multicolumn{7}{c}{ Frecuencia puntuación (\%) } \\
\hline Ítem & $M$ & $D T$ & 1 & 2 & 3 & 4 & $r^{\text {tot }}$ \\
Item 1 & 2.73 & .96 & 13.34 & 23.31 & 40.71 & 22.64 & .46 \\
Item 2 & 3.15 & .81 & 4.22 & 13.68 & 45.10 & 37.00 & .60 \\
Item 3 & 2.95 & .91 & 8.11 & 19.09 & 42.23 & 30.57 & .62 \\
Item 4 & 2.97 & .90 & 7.77 & 18.92 & 41.89 & 31.42 & .67 \\
Item 5 & 2.96 & .90 & 7.94 & 18.75 & 42.91 & 30.40 & .67 \\
Item 6 & 2.71 & .97 & 12.67 & 27.36 & 36.49 & 23.48 & .65 \\
Item 7 & 2.81 & .89 & 8.61 & 24.49 & 44.09 & 22.81 & .70 \\
Item 8 & 3.10 & .79 & 4.22 & 14.19 & 49.16 & 32.43 & .75 \\
Item 9 & 3.02 & .80 & 4.90 & 16.55 & 50.34 & 28.21 & .67 \\
Item 10 & 3.06 & .79 & 3.38 & 18.08 & 47.80 & 30.74 & .70 \\
$\alpha$ de Cronbach & \multicolumn{7}{c}{} \\
Media coeficiente correlación inter-ítem
\end{tabular}

Nota. Ver Tabla 3 para los nombres completos de los ítems.

media-alta $(51.52 \%)$ y tenían estudios primarios (53.88\%). Proporcionaban cuidados a su padre o madre $(37.33 \%)$, una mujer $(62.84 \%)$, con una edad media de 63.32 años $(D T=31.17)$ y que sufría de demencia $(30.07 \%)$. Cuidaban desde hacía 13.23 años $(D T=9.44)$ dedicando al cuidado 15.67 horas diarias $(D T=4.15)$.

Un $15.88 \%$ de los cuidadores presentó episodio depresivo mayor. La puntuación media de sintomatología depresiva fue 18.19 $(D T=11.37)$, de pensamientos automáticos negativos $49.66(D T=20.99)$, de neuroticismo 3.01 $(D T=1.95)$, de extraversión $3.65(D T=1.87)$ y de psicoticismo 1.26 (DT=.99) (ver Tabla 1).

No hubo diferencias significativas en las puntuaciones medias para las variables sociodemográficas y de la situación de cuidado, la autoeficacia, los síntomas depresivos, pensamientos automáticos negativos, rasgos de personalidad y diagnóstico de depresión entre las dos submuestras. No hubo diferencias significativas en la GSES en función de ninguna de las variables sociodemográficas o de la situación de cuidado, ni para la muestra total ni para los dos subgrupos.

\section{Análisis de fiabilidad}

La puntuación media de autoeficacia fue 29.45 ( $D T=6.29$, rango=10-40). Las medias de los ítems oscilaron entre 2.71 para el Ítem 6 y 3.15 para el Ítem 2. En relación con las respuestas de los sujetos, el porcentaje de los que puntuaron 1 fue del $7.52 \%$ (efecto suelo) y el porcentaje de aquellos que puntuaron 4 fue del $28.97 \%$ (efecto techo). Los coeficientes de correlación elementototal corregidos oscilaron entre .46 para el Ítem 1 y .75 para el Ítem 8 y todos fueron significativos $(p<.001)$. La media del coeficiente de correlación inter-ítem fue de .47 , con un mínimo de .29 y un máximo de .72. El alfa de Cronbach fue .90 (Tabla 2).

\section{Validez de constructo}

En relación al AFE, la prueba de Mardia (1970) no fue estadísticamente significativa para la asimetría $(p=1.00)$; sin embargo, hubo evidencia de excesiva curtosis $(p<.001)$, apoyando la decisión de emplear un procedimiento basado en correlaciones policóricas. El índice de KMO fue de $.91 \mathrm{y}$ el test de esfericidad de Bartlett de 1429.10 ( $p<.001)$. Los 10 ítems de la GSES se distribuyeron en un único factor, siendo la varianza total explicada por el mismo de $74.17 \%$. Las saturaciones factoriales de los ítems oscilaron entre .49 y .86 (ver Tabla 3 ).

Posteriormente, se realizó el AFC con el criterio de cuadrados mínimos ponderados. Los índices de ajuste fueron: $X^{2}{ }_{(35)}=202.45, p<.001$; $X^{2} / d f=5.79 ; \mathrm{SRMR}=.06 ; \mathrm{RMSEA}=.13 ; \mathrm{GFI}=.88$; AGFI=.81; NFI=.94; CFI=.95, indicando que el modelo tuvo un ajuste suficiente, aunque moderado, a los datos. La Tabla 4 muestra las cargas factoriales y errores de medida para cada ítem. Los pesos factoriales fueron satisfactorios, oscilando de .48 en el Ítem 1 a .68 en los Ítems 6 y 7. 
Tabla 3. Cargas Factoriales de los Ítems de la GSES (AFE)

Ítem

Factor 1

Puedo resolver la mayoría de los problemas si me esfuerzo lo necesario (8)

.86

$\mathrm{Al}$ tener que hacer frente a un problema, generalmente se me ocurren varias

alternativas de cómo resolverlo (10)

Si me encuentro en una situación difícil, generalmente se me ocurre qué debo hacer (9)

Venga lo que venga, por lo general soy capaz de manejarlo (7)

Tengo confianza en que podría manejar eficazmente acontecimientos inesperados (4)

Gracias a mis cualidades y recursos puedo superar situaciones imprevistas (5)

Cuando me encuentro en dificultades puedo permanecer tranquilo/a porque cuento con

las habilidades necesarias para manejar situaciones difíciles (6)

Me es fácil persistir en lo que me he propuesto hasta llegar a alcanzar mis metas (3)

Puedo resolver problemas difíciles si me esfuerzo lo suficiente (2)

Puedo encontrar la manera de obtener lo que quiero aunque alguien se me oponga (1) $\quad .49$

Nota. Se indica el número del ítem entre paréntesis.

Tabla 4. Cargas Factoriales y Errores de Medida para Cada ítem de la GSES (AFC)

\begin{tabular}{lcc}
\hline Ítem & $\lambda_{\mathbf{x}}$ & $\boldsymbol{\delta}$ \\
\hline Cuando me encuentro en dificultades puedo permanecer tranquilo/a porque cuento con & .68 & .50 \\
las habilidades necesarias para manejar situaciones difíciles (6) & .58 & .36 \\
Venga lo que venga, por lo general soy capaz de manejarlo (7) & .36 & .43 \\
Tengo confianza en que podría manejar eficazmente acontecimientos inesperados (4) & .66 & .66 \\
Gracias a mis cualidades y recursos puedo superar situaciones imprevistas (5) & .65 & .24 \\
Puedo resolver la mayoría de los problemas si me esfuerzo lo necesario (8) & .60 & .30 \\
Al tener que hacer frente a un problema, generalmente se me ocurren varias & .30 \\
alternativas de cómo resolverlo (10) & .32 \\
Si me encuentro en una situación difícil, generalmente se me ocurre qué debo hacer (9) & .60 & .55 \\
Me es fácil persistir en lo que me he propuesto hasta llegar a alcanzar mis metas (3) & .55 & .38 \\
Puedo resolver problemas difíciles si me esfuerzo lo suficiente (2) & .53 & .38 \\
Puedo encontrar la manera de obtener lo que quiero aunque alguien se me oponga (1) & .48 & .71 \\
\hline
\end{tabular}

Nota. Se indica el número del ítem entre paréntesis, $\lambda \mathrm{x}$ : carga factorial, $\delta$ : error de medida.

Con respecto a la validez convergente, se encontró una correlación inversa significativa entre neuroticismo y autoeficacia $(r=-.35, p<.001)$ y directa significativa entre extraversión y autoeficacia $(r=.36, p<.001)$. Por lo que respecta a la validez discriminante, no se encontró correlación entre psicoticismo y autoeficacia.

\section{Validez criterial}

En relación con la validez criterial, se encontraron correlaciones inversas significativas entre sintomatología depresiva y autoeficacia ( $r=-$ $.32, p<.001)$ y entre pensamientos automáticos negativos y autoeficacia $(r=-.27, p<.001)$. La $t$ de Student indicó que los cuidadores sin depresión presentaron puntuaciones más altas en autoeficacia que aquellos con depresión $(M=30.13$ vs. $M=25.84), t(119.64)=5.60, p<.001$.

El área bajo la curva ROC (AUC) fue .68
(95\% CI $\quad$.62 -.74). En consecuencia, la probabilidad de que la puntuación de la GSES clasifique correctamente a los cuidadores con y sin depresión es del 68\% (ver Figura 1). Con un punto de corte en la GSES de $\leq 28$ se tendría una sensibilidad del $71.00 \%$, una especificidad del $53.20 \%$, un VPP del $22.30 \%$ y un VPN del $90.70 \%$.

\section{Discusión}

El objetivo de este estudio fue analizar las propiedades psicométricas de la versión española de la GSES en cuidadores no profesionales.

Se obtuvo una media para la GSES de 29.45. Este dato es similar al 29.28 encontrado para la versión original por Schwarzer (1993) y está dentro del rango obtenido para 25 países por Scholz et al. (2002), que osciló entre 20.22 para 


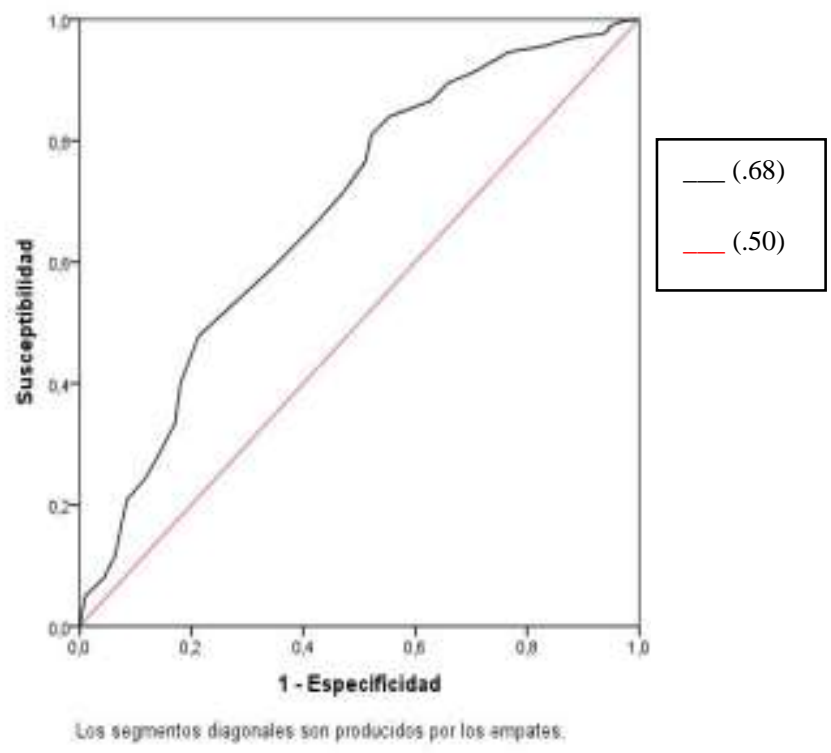

Figura 1. Curva operativa característica del receptor

los japoneses y 33.19 para los costarricenses (constituyendo esta última la media para la versión española de Baessler \& Schwarzer, 1996). Asimismo, esta puntuación fue superior a la encontrada en pacientes ambulatorios españoles (De las Cuevas \& Peñate, 2015), personas de baja laboral en Suecia (Löve et al., 2012), mujeres de Alemania y Polonia y mujeres trabajadoras de Costa Rica (Lusczynska et al., 2005). Similar a la hallada para adultos noruegos (Leganger et al., 2000). E inferior a la encontrada en el estudio de Lusczynska et al. (2005) para los hombres, las mujeres de Turquía y USA (independientemente de su ocupación) y las mujeres estudiantes de Costa Rica (Lusczynska et al., 2005); y en universitarios (Sanjuán et al., 2000) y adolescentes españoles (Espada et al., 2012); aunque las puntuaciones de estos dos últimos estudios no son directamente comparables, ya que utilizaron un rango de 10 a 100 para esta escala. Estas diferencias podrían explicarse parcialmente por cuestiones culturales, influyendo la cultura de procedencia en la percepción y afrontamiento de las dificultades (Crowther \& Austin, 2009).

La consistencia interna de la GSES fue excelente $(\alpha=.90)$, con una media del coeficiente de correlación inter-ítem de .47 y coeficientes de correlación elemento-total corregidos $\geq .46$. La consistencia interna fue superior a la encontrada para la muestra completa del estudio de Scholz et al. (2002) y para la submuestra de estudiantes universitarios costarricenses (Baessler \& Schwarzer, 1996); para trabajadores de Costa Rica, inmigrantes, profesores y estudiantes alemanes, estudiantes polacos, americanos y turcos (Lusczynska et al.,2005); adultos noruegos (Leganger et al., 2000) y estudiantes universitarios (Sanjuán et al., 2000), y adolescentes (Espada et al., 2012) españoles; y similar a la encontrada para estudiantes de Costa Rica (Lusczynska et al., 2005), personas en baja laboral suecas (Löve et al., 2012) y pacientes ambulatorios españoles (De las Cuevas \& Peñate, 2015). Asimismo, los coeficientes de correlación elemento-total y la media del coeficiente de correlación inter-ítem obtenidos satisfacen los criterios propuestos por Briggs \& Cheek (1986) para un adecuado equilibrio entre consistencia interna y amplitud de la medida.

El análisis factorial exploratorio identificó un único factor que explicó el $74.17 \%$ de la varianza total. Este hallazgo es coherente con la estructura unifactorial de la versión original (Schwarzer \& Jerusalem, 1995) y las validaciones en otras poblaciones (p.ej., Baessler \& Schwarzer, 1996; Espada et al., 2012; Scholz et al., 2002). Además, el análisis confirmatorio mostró un ajuste al modelo suficiente, aunque moderado.

Se encontró una correlación inversa de la GSES con neuroticismo y directa con extraversión. Estos datos indicarían que mayor autoeficacia se relaciona con una mayor estabilidad emocional, y coinciden con trabajos previos con universitarios (Schwarzer, 2014). Asimismo, se hallaron correlaciones inversas de la GSES con la sintomatología depresiva y los pensamientos automáticos negativos. El hallazgo de una correlación inversa con la sintomatología depresiva coincide con los resultados de Lusczynska et al. (2005) y los de Scholz et al. (2002) para hombres y mujeres de Costa Rica (correspondientes a la muestra de la versión española de Baessler \& Schwarzer, 1996); y contrasta con los datos de Espada et al. (2012), que no obtuvieron relación entre autoeficacia y sintomatología depresiva en adolescentes españoles. La correlación inversa entre la GSES y los pensamientos negativos iría en la línea de Semiatin \& O'Connor (2012) que establecen que aquellos con mayor autoeficacia tienen un pensamiento más positivo. 
El grado de concordancia entre los resultados de la GSES y el diagnóstico de depresión mayor es modesto ( $\mathrm{AUC}=.68$ ), pero razonable teniendo en cuenta que la GSES no evalúa sintomatología depresiva. Los datos sugieren que una puntuación de $\leq 28$ en autoeficacia es un punto de corte óptimo que discrimina entre cuidadores con o sin depresión, poniendo de relieve que es probable que los cuidadores con baja autoeficacia presenten depresión mayor. Futuros estudios deberían analizar si este punto de corte es aplicable a distintas poblaciones.

Este estudio presenta una serie de limitaciones. Entre ellas, el diseño transversal limita la posibilidad de establecer relaciones causales. Por otra parte, el carácter autoinformado de algunos instrumentos podría generar un sesgo en las respuestas, exacerbar la varianza común e incrementar artificialmente las correlaciones entre variables (Spector, 2006). Asimismo, un $1.82 \%$ de los cuidadores no cumplimentaron adecuadamente los cuestionarios y fueron eliminados del análisis, pudiendo introducir sesgos de no respuesta; sin embargo, dada la pequeña cantidad de cuestionarios eliminados, las repercusiones sobre los resultados serían mínimas. Además, futuros estudios deberían proporcionar información sobre la reproductibilidad y responsividad de la escala en esta población.

Los resultados permiten concluir que la GSES posee adecuadas propiedades psicométricas como medida de la autoeficacia en cuidadores, en línea con la versión original y apoyando la investigación internacional sobre la GSES en relación con la consistencia interna y la estructura factorial de la escala. Este instrumento puede ser empleado por investigadores y profesionales de la salud de manera fiable para evaluar la autoeficacia generalizada en cuidadores no profesionales.

\section{Agradecimientos}

Ministerio de Economía y Competitividad de España (Proyecto 2012/PN162 [PSI2012-37396]) y Unidad de Dependencia de la Dirección Xeral de Dependencia e Autonomía Persoal (Xunta de Galicia). Asimismo, también nuestro agradecimiento a la spin-off de la Universidad de Santiago de Compostela "Xuntos. Atención Psicológica y Psiquiátrica".

\section{Referencias}

Abad, F. J., Olea, J., Ponsoda, V., \& García, C. (2011). Medición en ciencias del comportamiento y de la salud. Madrid: Editorial Síntesis.

Baessler, J., \& Schwarzer, R. (1996). Evaluación de la autoeficacia: adaptación española de la escala de autoeficacia general. Ansiedad y Estrés, 2(1), 1-8.

Bandura, A. (1977). Self-efficacy: Toward a unifying theory of behavioral change. Psychological Review, 84(2), 191-215. doi:10.1037/0033-295X.84.2.191

Bandura, A., \& Wood, R. E. (1989). Effect of perceived controllability and performance standards on self-regulation of complex decision making. Journal of Personality and Social Psychology, 56(5), 805-814. doi:10.1037/0022-3514.56.5.805

Baumgartner, H., \& Homburg, C. (1996). Applications of structural equation modeling in marketing and consumer research: A review. International Journal of Research in Marketing, 13(2), 139-161. doi:10.1016/0167-8116(95)00038-0

Briggs, S. R., \& Cheek, J. M. (1986). The role of factor analysis in the development and evaluation of personality scales. Journal of Personality, 54(1), 106-148.

doi:10.1111/j.1467-6494.1986.tb00391.x

Cheng, S. T., Lam, L. C. W., Kwok, T., Ng, N. S. S., \& Fung, A. W.T. (2013). Self-efficacy is associated with less burden and more gains from behavioral problems of Alzheimer's disease in Hong Kong Chinese caregivers. Gerontologist, 53(1), 71-80. doi:10.1093/geront/gns062

Contador, I., Fernández-Calvo, B., Palenzuela, D. L., Miguéis, S., \& Ramos, F. (2012). Prediction of burden in family caregivers of patients with dementia: A perspective of optimism based on generalized expectancies of control. Aging \& Mental Health, 16(6), 675-682. doi:10.1080/13607863.2012.684666.

Crellin, N. E., Orrell, M., McDermott, O., \& Charlesworth, G. (2014). Self-efficacy and health-related quality of life in family carers of people with dementia: A systematic review. 
Aging \& Mental Health, 18(8), 954-969. doi:10.1080/13607863.2014.915921

Crowther, M., \& Austin, A. (2009). The cultural context of clinical work with aging caregivers. En S. H. Qualls, \& S. H., Zarit (Eds.), Aging families and caregiving (pp. 45-60). Hoboken, New Jersey: John Wiley \& Sons.

De Andrés-Jiménez, E., \& Limiñana-Gras, R. M. (2014). El afrontamiento del cuidado en familiares cuidadores de personas con demencia: Dimensiones cognitivas y el manejo de la ira. Revista Iberoamericana de Diagnóstico y Evaluación - e Avaliação Psicológica, 37(1), 169-185.

De las Cuevas, C., \& Peñate, W. (2015). Validation of the General Self-Efficacy Scale in psychiatric outpatient care. Psicothema, 27(4), 410-415. doi:10.7334/psicothema2015.56

Domínguez-Guedea, M. T. (2016). Bienestar en cuidadores familiares de adultos mayores: Un derecho, una aspiración y un constructo psicológico. Revista Iberoamericana de Diagnóstico y Evaluación Psicologica - e Avaliação Psicológica, 4l(1), 104-117.

Ducharme, F., Lévesque, L., Gendron, M., \& Legault, A. (2001). Development process and qualitative evaluation of a program to promote the mental health of family caregivers. Clinical Nursing Research, 10 (2), 182-201. doi:http://dx.doi.org/10.1177/10547730122158860

Espada, J. P., Gonzálvez, M. T., Orgilés, M., Carballo, J. L., \& Piqueras, J. A. (2012). Validación de la Escala de Autoeficacia General con adolescentes españoles. Electronic Journal of Research in Educational Psychology, 10(1), 355-370.

First, M. B., Williams, J. B. W., Karg, R. S., \& Spitzer, R. L. (2015). Structured Clinical Interview for DSM-5 Disorders, Clinician Version (SCID-5-CV). Arlington, VA: American Psychiatric Association.

Francis, L. J., Brown, L. B., \& Philipchalk, R. (1992). The development of an abbreviated form of the Revised Eysenck Personality Questionnaire (EPQR-A): its use among students in England, Canada, the USA and Australia. Personality and Individual Differences, 13(4), 443-449. doi:10.1016/0191-8869(92)90073-X
Gallagher, D., Mhaolain, A. N., Crosby, L., Ryan, D., Lacey, L., Coen, R. F, ... Lawlor, B. A. (2011). Determinants of the desire to institutionalize in Alzheimer's caregivers. American Journal of Alzheimer's Disease \& Other Dementias, 26(3), 205-211. doi:10.1177/1533317511400307

Hoffmann, F., \& Rodrigues, R. (2010). Informal carers: Who takes care of them? Viena: European Centre for Social Welfare Policy and Research.

Hogarty, K. Y., Hines, C. V., Kromrey, J. D., Ferron, J. M., \& Mumford, K. R. (2005). The quality of factor solutions in exploratory factor analysis: The influence of sample size, communality and overdetermination. Educational and Psychological Measurement, 65(2), 202-226. doi:10.1177/0013164404267287

Hollon, S. D., \& Kendall, P. C. (1980). Cognitive self-statements in depression: Development of an Automatic Thoughts Questionnaire. Cognitive Therapy and Research, 4(4), 383395. doi:10.1007/BF01178214

Hu, L., \& Bentler, P. M. (1999). Cutoff criteria for fit indexes in covariance structure analysis: Conventional criteria versus new alternatives. Structural Equation Modeling, 6(1), 1-55. doi:10.1080/10705519909540118

Hulley, S. B., Cummings, S. R., \& Newman, T. B. (2013). Designing cross-sectional and cohort studies. En S. B. Hulley, S. R. Cumming, W. S. Browner, D. G. Grady, \& T. B. Newman (Eds.), Designing Clinical Research, $4^{\text {th }} \mathrm{ed}$. (pp. 85-96). Philadelphia: Wolters Kluwer/ Lippincott Williams \& Wilkins.

Koerner, S. S., Kenyon, D. B., \& Shirai, Y. (2009). Caregiving for elder relatives: Which caregivers experience personal benefits/gains? Archives of Gerontology and Geriatrics, 48(2), 238-245. doi:10.1016/j.archger.2008.01.015

Leganger, A., Kraft, P., \& Røysamb, E. (2000). Perceived self-efficacy in health behaviour research: Conceptualisation, measurement and correlates. Psychology \& Health, 15(1), 5169. doi:10.1080/08870440008400288

Litzelman, K., Skinner. H. G., Gangnon, R. E., Nieto, F. J., Malecki, K., \& Witt, W. P. (2014). Role of global stress in the healthrelated quality of life of caregivers: Evidence from the Survey of the Health of Wisconsin. 
Quality of Life Research, 23(5), 1569-1578. doi:10.1007/s11136-013-0598-z

Lloret-Segura, S., Ferreres-Traver, A., HernándezBaeza, A., \& Tomás-Marco, I. (2014). El análisis factorial exploratorio de los ítems: Una guía práctica, revisada y actualizada. Anales de Psicología, 30(3), 1151-1169. doi:10.6018/analesps.30.3.199361

Lopez, J., Romero-Moreno, R., MárquezGonzález, M., \& Losada, A. (2012). Spirituality and self-efficacy in dementia family caregiving: Trust in god and yourself. International Psychogeriatrics, 24(12), 19431952. doi:10.1017/ S1041610212001287

Lorenzo-Seva, U., \& Ferrando, P. J. (2006). FACTOR: A computer program to fit the exploratory factor analysis model. Behavior Research Methods, 38(1), 88-91. doi:10.3758/BF03192753

Lorenzo-Seva, U., \& Ferrando, P. J. (2012). Manual of the Program FACTOR. Recuperado de http://psico.fcep.urv.es/utilitats/factor/

Löve, J., Moore, C. D., \& Hensing, G. (2012). Validation of the Swedish translation of the General Self-Efficacy Scale. Quality of Life Research, 21(7), 1249-1253. doi:10.1007/ s11136-011-0030-5

Luszczynska, A., Gutiérrez-Doña, B., \& Schwarzer, R. (2005). General self-efficacy in various domains of human functioning: Evidence from five countries. International Journal of Psychology, 40(2), 80-89. doi:10.1080/00207590444000041

Mardia, K. V. (1970). Measures of multivariate skewnees and kurtosis with applications. Biometrika, 57(3), 519-530. doi:10.2307/2334770

Otero, P., Vázquez, F. L., Blanco, V., \& Torres, A. (2017). Propiedades psicométricas del "Cuestionario de Pensamientos Automáticos" (ATQ) en cuidadores familiares. Behavioral Psychology, 25(2), 387-403.

Pearlin, L. I., Mullan, J. T., Semple, S. J., \& Skaff, M. M. (1990). Caregiving and the stress process: An overview of concepts and their measures. Gerontologist, 30(5), 583-594. doi:10.1093/geront/30.5.583

Radloff, L. S. (1977). The CES-D Scale: A selfreport depression scale for research in the general population. Applied Psychological
Measurement,

$1(3)$,

$385-401$.

doi:10.1177/014662167700100306

Sandín, B., Valiente, R. M., Chorot, P., Olmedo, M., \& Santed, M. A. (2002). Versión española del cuestionario EPQR-Abreviado (EPQR-A) (I): Análisis exploratorio de la estructura factorial. Revista de Psicopatología y Psicología Clínica, 7(3), 195-205. doi:10.5944/rppc.vol.7.num.3.2002.3933

Sandín, B., Valiente, R. M., Olmedo, M., Chorot, P., \& Santed, M. A. (2002). Versión española del cuestionario EPQR-Abreviado (EPQR-A) (II): replicación factorial, fiabilidad y validez. Revista de Psicopatología y Psicología Clínica, 7(3), 207-216. doi:10.5944/rppc.vol.7.num.3.2002.3934

Sanjuán, P., Pérez, A. M., \& Bermúdez, J. (2000). Escala de autoeficacia general: Datos psicométricos de la adaptación para población española. Psicothema, 12(Suppl 2), 509-513.

Savundranayagam, M. Y., Montgomery, R. J. V., Kosloski, K., \& Little, T. D. (2011). Impact of a pscyhoeducational program on three types of caregiver burden among spouses. International Journal of Geriatric Psychiatry, 26(4), 388-396. doi:10.1002/gps.2538

Scholz, U., Doña, B., Sud, S., \& Schwarzer, R. (2002). Is general self-efficacy a universal construct? Psychometric findings from 25 countries. European Journal of Psychological Assessment, 18(3), 242-251. doi:10.1027//1015-5759.18.3.242

Schwarzer, R. (1993). Measurement of perceived self-efficacy. Psychometric scales for crosscultural research. Berlin: Freien Universität Berlin.

Schwarzer, R. (2014). Everything you wanted to know about the General Self-Efficacy Scale but were afraid to ask. Recuperado de: http://userpage.fu-

berlin.de/\%7Ehealth/faq_gse.pdf

Schwarzer, R., \& Jerusalem, M. (1995). Generalized Self-Efficacy Scale. En J. Weinman, S. Wright, \& M. Johnston (Eds.), Measures in health psychology: A user's portfolio. Causal and control beliefs (pp. 3537). Windsor: Nfer-Nelson.

Semiatin, A. M., \& O'Connor, M. K. (2012). The relationship between self-efficacy and positive aspects of caregiver in Alzheimer's disease 
caregivers. Aging \& Mental Health, 16(6), 683-688. doi:10.1080/13607863.2011.651437.

Spector, P. E. (2006). Method variance in organizational research: Truth or urban legend? Organizational Research Methods, 9(2), 221-232. doi:10.1177/1094428105284955

Stansfeld, J., Stoner, C. R., Wenborn, J., Vernooij-Dassen, M., Moniz-Cook, E., \& Orrell, M. (2017). Positive psychology outcome measures for family caregivers of people living with dementia: A systematic review. International Psychogeriatrics, 29(8), 1281-1296. doi:10.1017/S1041610217000655

Timmerman, M. E., \& Lorenzo-Seva, U. (2011). Dimensionality Assessment of Ordered Polytomous Items with Parallel Analysis. Psychological Methods, 16(2), 209-220. doi:10.1037/a0023353

Torres, A., Blanco, V., Vázquez, F. L., Díaz, O., Otero, P., \& Hermida, E. (2015). Prevalence of major depressive episodes in nonprofessional caregivers. Psychiatry Research, 226(1), 333-339.

doi:10.1016/j.psychres.2014.12.066

Vázquez, F. L., Blanco, V., \& López, M. (2007). An adaptation of the Center for Epidemiologic Studies Depression Scale for use in nonpsychiatric Spanish populations. Psychiatry Research, 149(1-3), 247-252. doi:10.1016/ j.psychres.2006.03.004

World Health Organization [WHO]. (2015). World report on aging and health. Geneva: World Health Organization. 